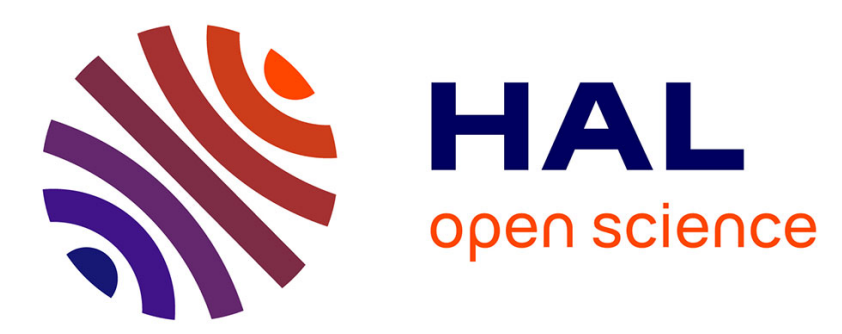

\title{
Perspectives offertes par les cellules solaires en couches minces pour les applications spatiales \\ P. Vasseur
}

\section{To cite this version:}

P. Vasseur. Perspectives offertes par les cellules solaires en couches minces pour les applications spatiales. Revue de Physique Appliquée, 1966, 1 (3), pp.228-230. 10.1051/rphysap:0196600103022800 . jpa-00242722

\section{HAL Id: jpa-00242722 https://hal.science/jpa-00242722}

Submitted on 1 Jan 1966

HAL is a multi-disciplinary open access archive for the deposit and dissemination of scientific research documents, whether they are published or not. The documents may come from teaching and research institutions in France or abroad, or from public or private research centers.
L'archive ouverte pluridisciplinaire HAL, est destinée au dépôt et à la diffusion de documents scientifiques de niveau recherche, publiés ou non, émanant des établissements d'enseignement et de recherche français ou étrangers, des laboratoires publics ou privés. 


\title{
PERSPECTIVES OFFERTES PAR LES CELLULES SOLAIRES EN COUCHES MINCES POUR LES APPLICATIONS SPATIALES
}

\author{
Par P. VASSEUR, \\ Centre National d'Études Spatiales, Brétigny.
}

\begin{abstract}
Résumé. - La comparaison entre les différentes sources de puissance à bord des satellites, utilisant l'énergie solaire, fait apparaître un net avantage aux cellules solaires en couches minces de CdS ou de CdTe.

La puissance spécifique élevée, 200 à $300 \mathrm{~W} / \mathrm{kg}$, leur bonne tenue sous rayonnement, leur flexibilité sont leurs qualités dominantes. Cependant, il reste à résoudre le problème capital de leur vieillissement.

Abstract. - Comparison between different power sources using solar energy aboard satellites shows the superiority of thin film CdS and CdTe solar cells.

Their outstanding qualities are : high specific energy, 200 to $300 \mathrm{~W} / \mathrm{kg}$, good performance when exposed to radiation and their flexibility.

However the important problem of ageing has still to be solved.
\end{abstract}

Parmi les différentes sources d'énergie utilisables à bord des véhicules spatiaux, l'énergie solaire occupe une place à part. Cette énergie, inépuisable, dont le spectre s'étend d'environ 0,3 à 1,4 micron, est disponible dans l'espace intersidéral avec une densité de $1,4 \mathrm{~kW} / \mathrm{cm}^{2}$ au voisinage de la terre.

Pour les missions dont la durée est supérieure au mois, et pour des puissances ne dépassant pas quelques dizaines de $\mathrm{kW}$ électriques, elle présente des avantages indiscutables : puissance spécifique faible du générateur, conversion statique possible, durée de fonctionnement uniquement limitée par la fiabilité du système de conversion.

Par contre son utilisation présente quelques servitudes : sa directivité impose soit une orientation du générateur vers le soleil, soit un générateur surabondant; les cycles d'illumination et d'obscurité dans le cas des satellites circumterrestres grèvent le bilan de poids par la nécessité d'avoir une batterie d'accumulateurs ; la variation de la densité de puissance en fonction de la distance au soleil, limite son emploi dans le cas des sondes lointaines que leur missions dirigent soit vers le soleil, soit vers les planètes les plus éloignées du soleil.

Environ 400 satellites actuellement lancés tirent leur puissance électrique de la conversion directe de l'énergie solaire par effet photovoltaïque. D'autres systèmes de conversion, passifs ou dynamiques, peuvent être utilisés en concentrant l'énergie solaire.

Pour comparer les différents systèmes de conversion applicables à la recherche spatiale il faut rechercher les critères homogènes, j'en choisirai deux :

- la fiabilité dont l'importance croît avec la durée de la mission;

- la puissance spécifique qui doit être aussi faible que possible.
Toutefois, dans le cas de ce deuxième critère, il faut prendre quelques précautions pour obtenir des valeurs comparables. Pour éviter toute confusion j'éliminerai de ce bilan de poids, d'une part les systèmes électroniques de régulation qui suivent les convertisseurs, d'autre part la batterie d'accumulateurs des satellites circumterrestres.

De plus, il est nécessaire de choisir le cas où le générateur est placé face au soleil, étant entendu que pour toute autre orientation on applique un coefficient d'aspect pour le calcul de la puissance.

Partant de ces bases, je vais faire une comparaison des différents systèmes de conversion directe et aussi tenter d'y placer les cellules en couches minces avec les résultats obtenus à ce jour.

1. Conversion statique avec concentrateur de lumière. - Les cellules thermo-photovoltaïques du type P I N au germanium permettent la conversion directe avec un flux lumineux de l'ordre de $25 \mathrm{~W} / \mathrm{cm}^{2}$; pour obtenir, dans l'espace, cette densité de puissance, il faut faire une concentration avec un facteur de l'ordre de 200. Le concentrateur doit permettre d'obtenir une répartition d'énergie aussi uniforme que possible sur les éléments actifs.

Le maintient d'une température admissible pour les éléments du convertisseur nécessite leur refroidissement en dissipant la chaleur non convertie dans un radiateur. En supposant un rendement de conversion de $20 \%$, il faut dissiper, par mètre carré de concentration, une puissance de $1120 \mathrm{~W}$, ce qui nécessite une surface de radiation de l'ordre de $0,5 \mathrm{~m}^{2}$

La réalisation d'un tel concentrateur est possible avec des éléments légers (structures en nid d'abeilles) Toutefois, si on veut une répartition d'énergie sur 
les cellules thermo-photovoltaïques, il est nécessaire d'avoir un miroir de surface relativement bien définie.

Il devrait être possible d'obtenir, par ce procédé, une puissance spécifique de l'ordre de $50 \mathrm{~W} / \mathrm{kg}$. Toutefois le fonctionnement, à température élevée, de la diode risque d'en diminuer la fiabilité. L'inconvénient majeur de ce système est la nécessité de pointer le concentrateur vers le soleil avec une bonne précision. Son utilisation spatiale sera vraisemblablement liée à des missions très particulières comme par exemple l'étude du rayonnement solaire au voisinage du soleil.

\section{Conversion par effet photovoltaïque.}

Le silicium. - Les premières jonctions photovoltaïques ont été réalisées au moyen de jonction $n / p$ d'une surface de $2 \mathrm{~cm}^{2}$ de et l'ordre de $300 \mu$ d'épaisseur. L'électrode postérieure est réalisée par dépôt sous vide d'aluminium, puis par nickelage. Pour l'électrode antérieure, on réalise un peigne, sous ce peigne on dépose une couche antiréfléchissante de SiO. Pour protéger partiellement la jonction contre les rayonnements qui diminuent la durée de vie des porteurs minoritaires dans la jonction, on place devant la cellule une lame de verre de 0,2 à $0,7 \mathrm{~mm}$ d'épaisseur. Enfin, pour limiter l'échauffement des cellules, on dépose un filtre interférentiel qui réfléchit les photons d'énergie extérieure au domaine spectral de sensibilité de la jonction.

Les rendements de ces cellules sont de $14 \%$. Le poids d'une telle cellule est de $0,35 \mathrm{~g}$ à $0,25 \mathrm{~g}$, suivant l'épaisseur de la fenêtre de protection. La rigidité de ces cellules oblige à les coller, pour les assembler sur des panneaux de structures en nid d'abeilles $\left(0,30 \mathrm{~g} / \mathrm{cm}^{2}\right)$. Si on accepte un rendement de $14 \%$, on obtient une puissance spécifique de l'ordre de $40 \mathrm{~W} / \mathrm{kg}$. Toutefois, il faut tenir compte de la perte de rendement due aux rayonnements, par exemple, pour une orbite polaire à $15000 \mathrm{~km}$, la perte de rendement est de $25 \%$ au bout de trois mois avec une fenêtre de verre de 4 mils d'épaisseur.

Il semble possible d'augmenter cette puissance spécifique, d'une part, en améliorant les caractéristiques de la jonction : il est possible, sans diminuer le rendement, d'abaisser l'épaisseur à $100 \mu$, et en réalisant une jonction à gradient de concentration, d'augmenter la tenue sous irradiation; enfin, d'autre part, en réalisant des structures en nid d'abeilles de béryllium, de diminuer le poids du support en amincissant la jonction.

Il semble donc possible d'atteindre la valeur de $50 \mathrm{~W} / \mathrm{kg}$ avec de telles cellules.

Les couches minces. - Au cours des conférences de P. Rappaport, F. Shirland, D. A. Cusano, nous ont été décrites les différentes cellules en

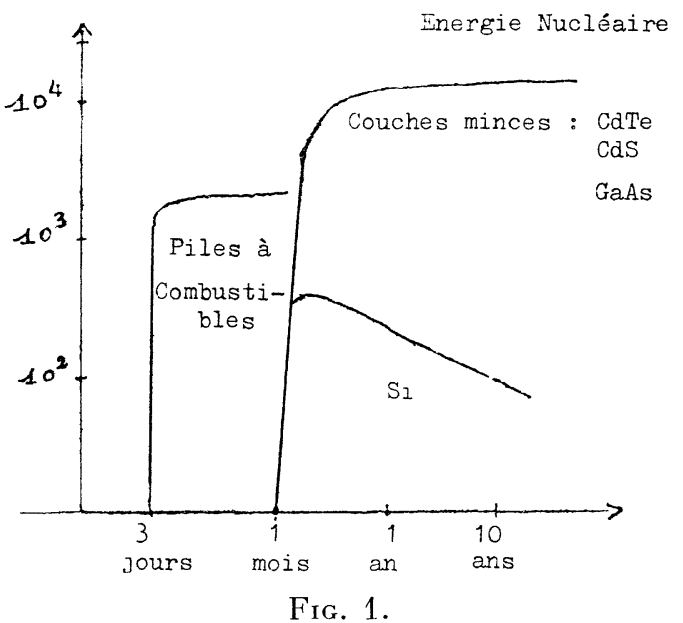

couches minces de Ga As, de CdS de Cd Te déposées sur des feuilles de molybdène, d'aluminium ou de matière plastique.

Les principales caractéristiques de ces cellules actuellement atteintes sont : la légèreté, et la souplesse dues à la faible épaisseur, la bonne résistance aux rayonnements.

Les rendements obtenus sont voisins de $5 \%$, mais il reste à améliorer leur stabilité dans le temps.

En prenant les mêmes hypothèses que celles qui ont été choisies pour les cellules au silicium, un rapide calcul donne :

- pour des cellules de CdS sur support plastique avec un rendement de $4 \%$, une puissance spécifique de $350 \mathrm{~W} / \mathrm{kg}$;

- pour des cellules de Cd Te sur support de molybdène avec un rendement de $5 \%$, une puissance spécifique de $150 \mathrm{~W} / \mathrm{kg}$;

- pour des cellules de Ga As, avec un rendement de $10 \%$, une valeur de $200 \mathrm{~W} / \mathrm{kg}$ a été indiquée.

Ces valeurs n'auront leur intérêt pour les applications spatiales que si un certain nombre de problèmes technologiques sont résolus :

- fabrication en série et agencement des cellules élémentaires sur des supports souples ;

- réalisation de structures déployables (par exemple par gonflage) ;

- étude et obtention de matières plastiques thermostables comme les déphényléthers (film $\mathrm{H}$ de la Dupont de Nemours), ayant de plus une très bonne résistance aux rayonnements.

A titre d'exemple, avec une sphère de 30 mètres de diamètre recouverte de cellules au CdS d'un rendement de $4 \%$, il est théoriquement possible d'obtenir une puissance électrique de l'ordre de $20 \mathrm{~kW}$

Des structures planes déployables par gonflage sont réalisables et permettront de réaliser des générateurs solaires de $20 \mathrm{~kW}$ et dont le poids variera de 100 à $150 \mathrm{~kg}$. 
Conclusions. - En rassemblant sur le diagramme puissance, durée de vie les résultats décrits précédemment, on constate que les photopiles en couches minces devraient prendre leur place, au-dessus du domaine des cellules photovoltaïques au silicium, et repousser vers des puissances supérieures à quelques dizaines de kilowatts, l'utilisation de l'énergie nucléaire.

Dans l'état actuel de la technologie de ces cellules, il n'est pas possible de choisir parmi les semiconducteurs actuellement étudiés.

Ce choix ne pourra être fait qu'après un programme de recherches fondamentales et technolo- giques dont les principaux chapitres pourraient être :

- l'étude de la tenue aux rayonnements ;

- l'étude de cellules transparentes au rayonnement infrarouge et réfléchissantes dans l'ultraviolet ;

- l'étude de matières plastiques thermostables ; - l'étude des jonctions entre les cellules élémentaires et des techniques d'assemblage ;

- l'étude de semiconducteurs tels que P I N.

Cet effort, nécessaire à la qualification spatiale des cellules en couche mince, devra être fait pendant au moins 3 ou 4 ans, pendant lesquels les cellules au silicium seront encore seules utilisées. 